\title{
Variable speed control for 2Ph-HSM in RGS: a comparative simulation study
}

Salam Waley Shneen, Mohammed Qasim Sulttan, Manal Hadi Jaber

Energy and Renewable Energies Technology Center, University of Technology, Iraq

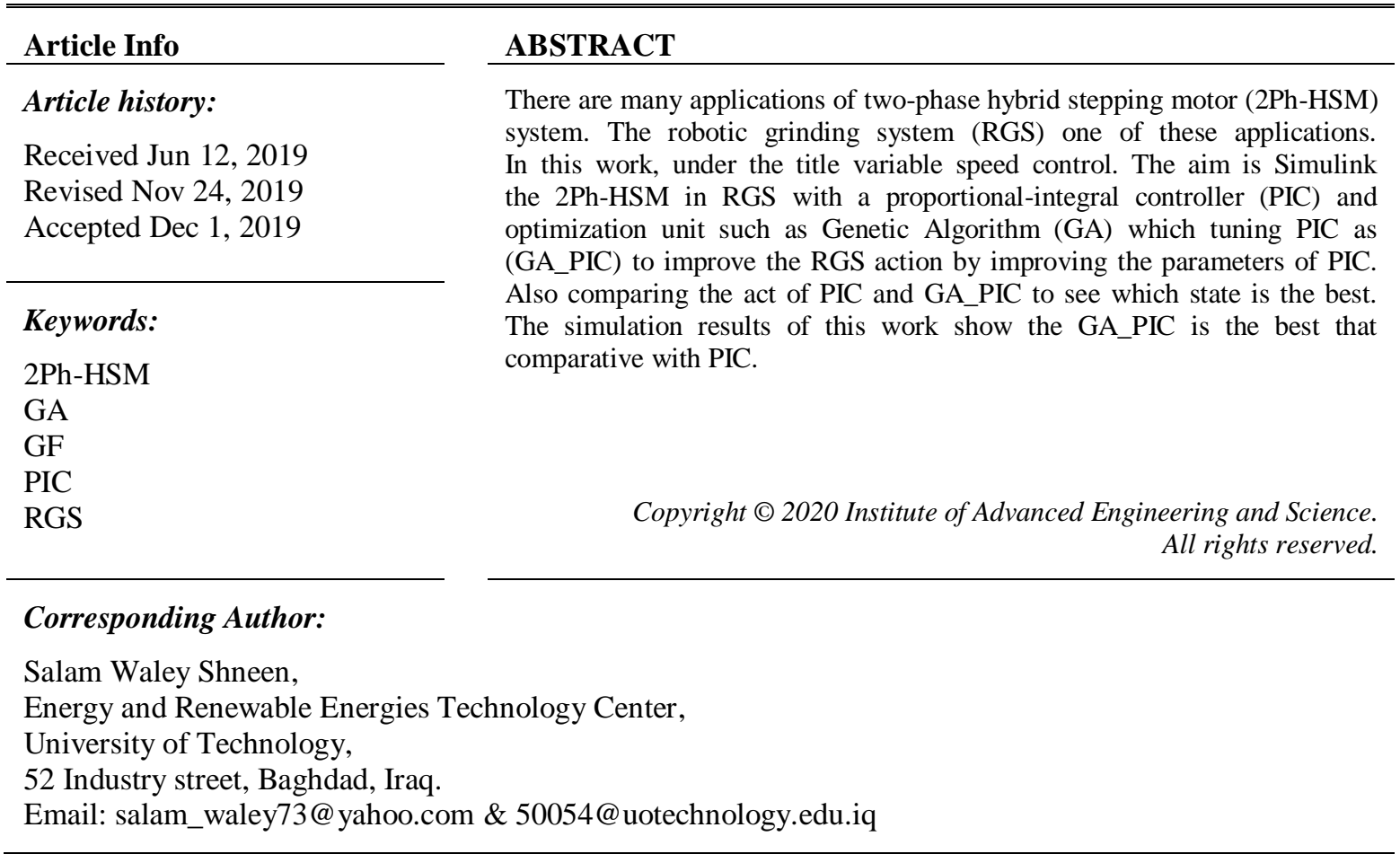

\section{INTRODUCTION}

The two-phase hybrid stepping motor (2Ph-HSM) system one of the machine drive system (MDS) that can be using in robotic grinding system (RGS) [1]. The proportional-integral controller (PIC) [2, 3] one of the classical controllers that can be using with MDS. The Genetic Algorithm (GA) [4-6] one of the optimal controller system that can be using with MDS [7]. This work uses Matlab to simulate the RGS including, 2Ph-HSM, control unit PIC and optimal controller system GA. RGS is a promising technicality to generate the final form of the required design. It can keep the human from taking part in noisy and dirty environments, get better product quality, and slash production expenses [8]. Some fieldwork formerly showed a difficulty in grinding the complicated form in workpiece like a marine propeller or turbine blade which requires the end-effector of a manipulator to hold over a stable connect force with the environment while the grinding tool moving along the workpiece. To carry out such a job and at the one time, both the strength and location of the robot should be controlled. Those control systems were classified into two major strategies, hybrid control for the force/position, and impedance control. Those strategies need a careful dynamic pattern of the manipulator and interaction of contact strength. In a strategy of a hybrid control for force/position, only natural trend path is undergoing a constant pressure or strength control, and this has some advantages like, the first is the dimensional change is compensated automatically because of the tool corrosion, and second is the accuracy on the planned path is relaxed since the confirmed connect from the strength control loop will offset the program error [9].

The PIC is a conventional controller and commonly used in many industrial processes. In the practical field, the hardware systems have non-controllable properties like the high order and non-linearity, so the good methods of PI tuning are more suitable from other methods. The PIC performance can be determined by many methods such as trial and error method, Ziegler-Nichols numeral method and the development mechanisms 
based on searching. The numerical worth of the PI controller profits counts on the estimates of the motor [10]. The Ziegler- Nichols method provided a methodical tuning to the PI parameters Which is characterized by a good reduction in pregnancy disorder, but with a long time for stability and great overshoot, it exhibits frustrating performance. To improve the performance of such systems, many studies are seeking to merge features on the footing of the experiences of particularist with consideration to PI gain, and the utilize of GA appears to be especially proper [11]. The implementation of modern optimization techniques such as GAs, it is a technique of searching best solutions at a sensible reckoning cost without being able to warranty either probability or optimality, or even in many cases to clarify how close to optimality an especially suitable solution [12].

In [13] the authors did a comparison between a classic PID controller and the Fuzzy-PID controller and they are got good results when reducing the value of setting time and the maximum overshoot value which mean that the performance of the fuzzy-PID controller is best than classic PID algorithm. In [14], the work achieved good performance when used PSO-PI controller than the Fuzzy-PI controller and traditional PI controller to control to robotic Grinding Force (GF) with use the 2-ph hybrid stepping motor. the authors used Matlab/Simulink to simulate the methods of control. The authors in a paper [15] used some intelligent methods to optimizing the PID controller such as ANN, ANFIS, and GA. The simulation results have appeared a good performance for intelligent methods than the classic $\mathrm{ZN}$ method in terms of different performance properties. The paper [16] shows the importance and effect of GAs to optimizing of control processes, also discusses the connotation and design execution of GA as an optimization gadget. The authors used the Matlab/Simulink to execute the models of this work. The simulation results showed a good optimization of GA controllers than fuzzy logic and classical controllers. The work [17] used two techniques to evaluate the performance of the PID controller, the differential evolution DE and GA. Two methods used to evaluate DE and GA, mean square error (MSE) and integral absolute error (IAE). The work achieved a good comparison between the performance of the GA-PID controller and DE-PID controller with the Ziegler-Nichols method.

In this work, the PIC is utilized as the control unit to controlling to RGS work and utilize from GA to tune the parameters of PIC as GA_PIC to optimizing the act of control unit which controls in 2Ph-HSM and GF. The rest of the paper is organized as follows: in section 2 the description of GF controlling in RGS. Section 3 illustrates the 2Ph-HSM in RGS into two parts, the mathematical and simulation model of the 2Ph-HSM system respectively. The controlling and optimization of the plant is clarified in section 4 by two sides the PIC and the GA_PIC optimization in 4.1 and 4.2 respectively. The simulation of results is discussed in section 5. Section 6 includes conclusion and future works.

\section{THE GF CONTROLLING IN RGS}

The GF decompose into three component forces, Normal GF (fN), Tangential GF (fT), and the third force is not significant which is work in the same direction of the Feed Direction (Fd), so it is neglected and this shown in Figure 1. The fT is mainly affecting on the consuming of power and preserve the lifetime of grinding wheel. The $\mathrm{fN}$ influence on the deformity of the surfaces and the roughness of surfaces [18]. Two sections will compose the Compacting Force (fC), the 2Ph-HSM and a Helix Spring (HS), the machining surface as a negative congruence. The 2ph-HSM is a brushless DC electric motor which splits a total rotation to a digit of equal steps [19]. The place of the motor can thereafter be ruled to stir and curb without any feedback sensor at one of the steps of rotation, while the motor is guardedly sized to the enforcement. The 2ph-HSM is used to control to controlling instruments with its fittings of high delicacy, less noise, less vibration, cheap cost, and a rising torque in output [20]. The GF is a mechanical process used to remove rough and very thin layers of articles on the extrinsic border of the workpiece.

The SM is rapid and executable in many execution hardware. The benefit of SM has increased due to its best reliability which comes from the elimination of mechanical brushes, and it spins slowly because it has a higher torque-to-inertia ratio, and also has a better heat squandering because of the windings are existing on the stator, not the rotor. The SM has a stepping ability that makes it take a good positioning without feedback, while when using closed-loop control to SM make it achieve fast response times and greater resolution capabilities. The SM designed to be performed in open-loop, and it can work at high velocity if take into consideration the nonlinear impacts [21]. When the SM work, it starts to compress on the HS provided the fC for a force control system, the $\mathrm{fC}$ is revealed by two force sensors constitute two orthogonal angles $\beta$ and $\alpha$. According to the data come from the sensors, the linear SM offset is changed and cause a compress on HS to modify the fN, this can be shown in Figure 2. The controlling of GF is based on the strategy of hybrid movement-force control when the $\mathrm{fN}$ is the surroundings force required, (fM) is referring to the Modifying Force that changes the adapting the controlling to the force at the needed. 


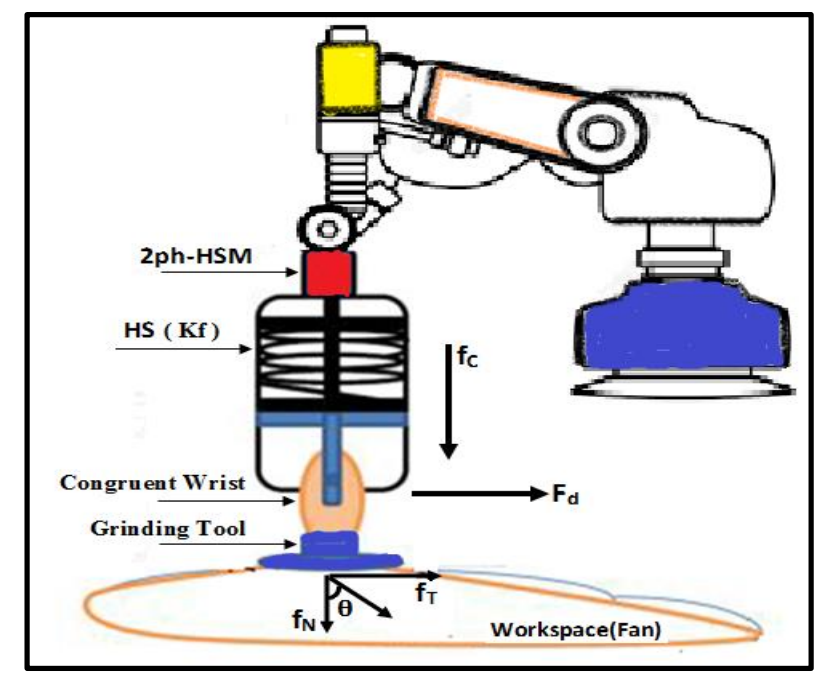

Figure 1.Model of the GF servo system

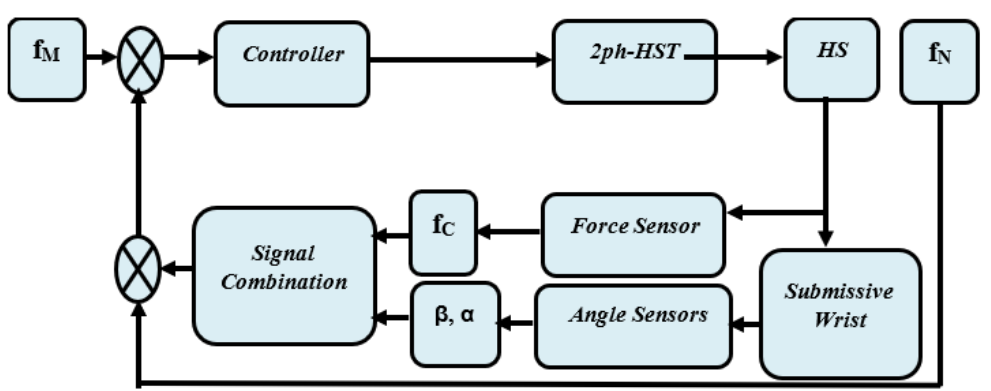

Figure 2. Schematic diagram of a force control system

The force control system is designed as following [22]:

$$
\begin{aligned}
& \mathrm{dp}=\frac{\mathrm{dg}+\mathrm{ds}}{2} \\
& \mathrm{f}_{\mathrm{N}}=\frac{\mathrm{Kf}(\mathrm{dg}+\mathrm{ds})}{2} \\
& \mathrm{f}_{\mathrm{T}}=\rho \mathrm{f}_{\mathrm{N}} \\
& \rho=\tan \theta
\end{aligned}
$$

where:

$\mathrm{dp} \quad:$ is the precise displacement of the SM at the GF with its coveted value $\mathrm{fN}$,

$\mathrm{dg} \quad:$ is the SM greatest displacement,

ds : is the smallest displacement to compress the HS,

Kf $\quad$ : is the elastic modulus of the HS,

Ft $\quad:$ is the Tangential force (set value per unit width), and

$\rho \quad$ : is the GF ratio

\section{THE 2Ph-HSM IN RGS}

In this part, the parameters of the $2 \mathrm{Ph}-\mathrm{HSM}$ that can be clearly by the model system, the equations and the simulation model for 2Ph-HSM system as the following: 


\subsection{Mathematical Model for 2Ph-HSM system}

The 2Ph-HSM system one of the machine drive system (MDS) include two systems the first system is the electrical and the second is the mechanical system. The electrical system includes the voltage in volt (v), current in ampere (A), resistance in $(\Omega)$ ohm and inductor in Henry $(\mathrm{H})$. Figure 3 shown the equivalent circuit of HSM. The mechanical system includes the motor torque, friction torque in N.m, rotational inertia, viscous friction, motor speed and shaft power [23].

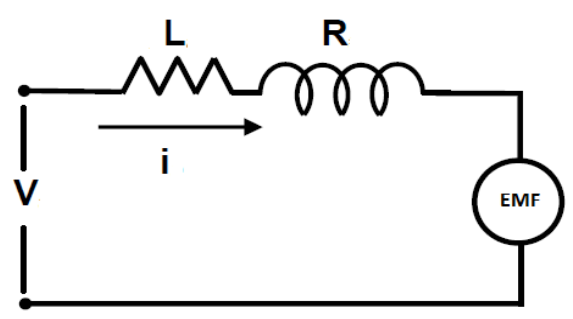

Figure 3. The equivalent circuit of HSM

The Transfer Function (T.F) G(s) for 2Ph-HSM of an open-loop system is calculated as in [24]:

$$
\mathrm{G}(\mathrm{s})=\mathrm{A}(\mathrm{s}) / \mathrm{B} 1(\mathrm{~s})+\mathrm{B} 2(\mathrm{~s})
$$

\subsection{Simulation Model for 2Ph-HSM system}

This section appears the simulation model of a 2Ph-HSM system. Figure 4 (a) shown the simulation model without the controller by using constant speed which includes the T.F of RGS, the input system and the output system. Figure 4 (b) shown the simulation model without the controller by using variable speed which includes the T.F of RGS, the input system and the output system. Figure 5 (a and b). Shown the simulation model with the PIC by using constant speed and variable speed respectively includes the T.F of RGS, PIC, the input and output systems.

The simulation model of a 2Ph-HSM system with an optimum system GA_PIC includes the T.F of RGS, GA_PIC the input system and the output system is the same model as in Figure 5 (a and b) with an essential modification by changing the PIC unit by GA_PIC unit. The simulation model of a 2Ph-HSM system with an optimum system is shown in Figure 6. Figure 7 shown the simulation model of the 2Ph-HSM system combined for three circuits (without control, with PIC, and with GA_PIC) for constant speed as an input speed to make the comparison between the output results of these three circuits. The simulation model of a $2 \mathrm{Ph}-\mathrm{HSM}$ system combined for three circuits (without control, with PIC, and with GA_PIC) and for variable speed appears in Figure 8.

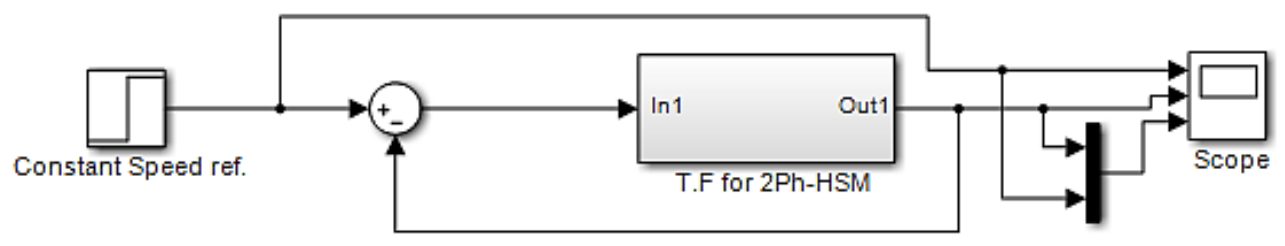

a. Constant Speed Ref. i/p

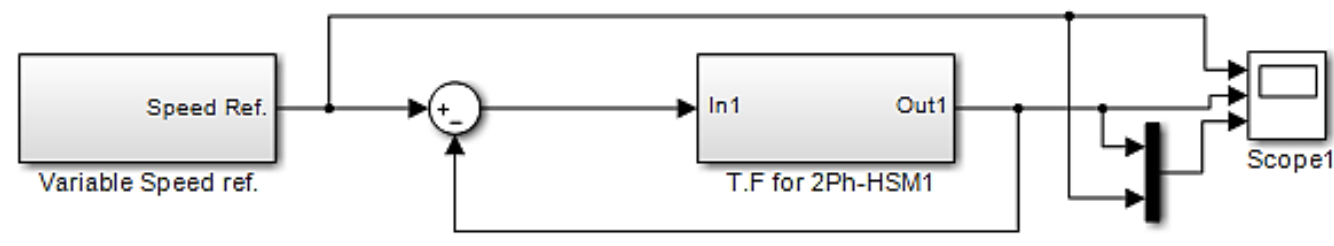

b. Virable Speed Ref. i/p

Figure 4. The simulation model of $2 \mathrm{Ph}-\mathrm{HSM}$ system without controller 


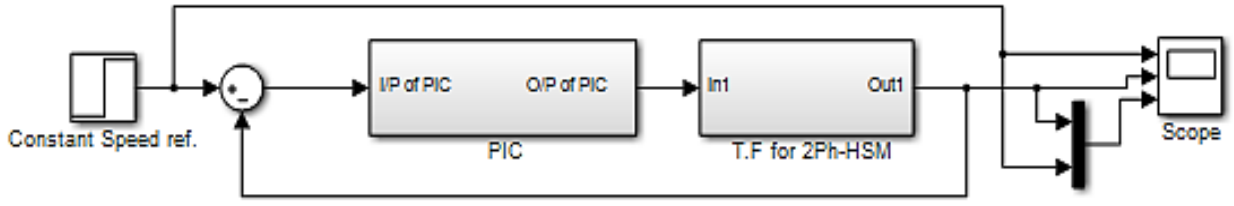

a. Constant Speed Ref. i/p

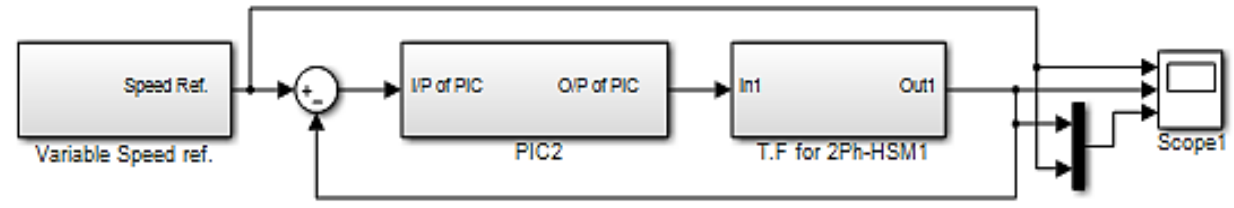

b. Virable Speed Ref. ip

Figure 5. The simulation model of 2Ph-HSM system with PIC

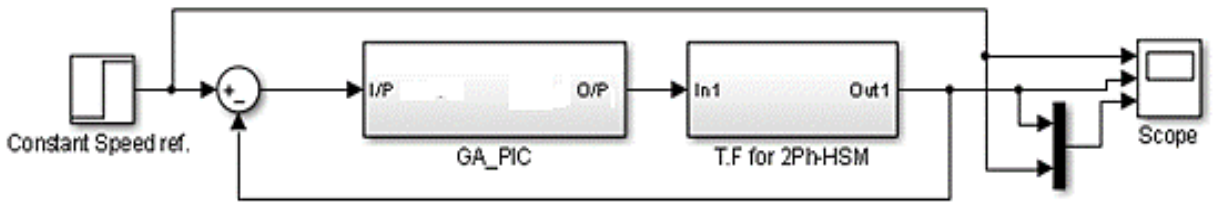

a Constant Speed Ref. ip

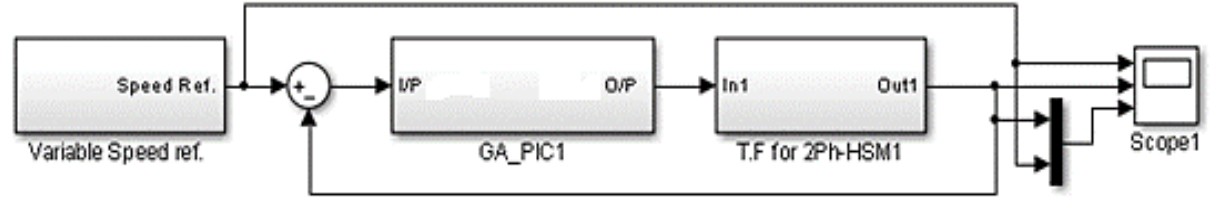

b. Virable Speed Rer. ip

Figure 6. The simulation model of 2Ph-HSM system with GA_PIC

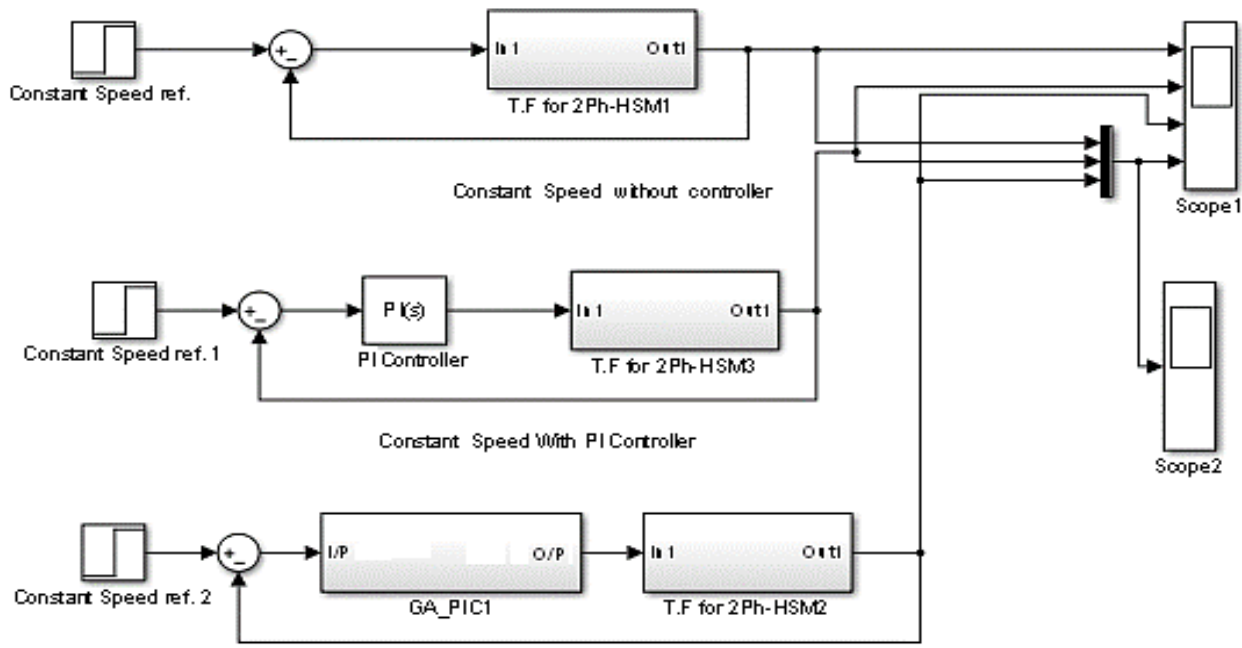

Constart Speed Wutth GA_PI Controller

Figure 7. The simulation model of a 2Ph-HSM system combined for three circuits (without control, with PIC, and with GA_PIC) and for constant speed 


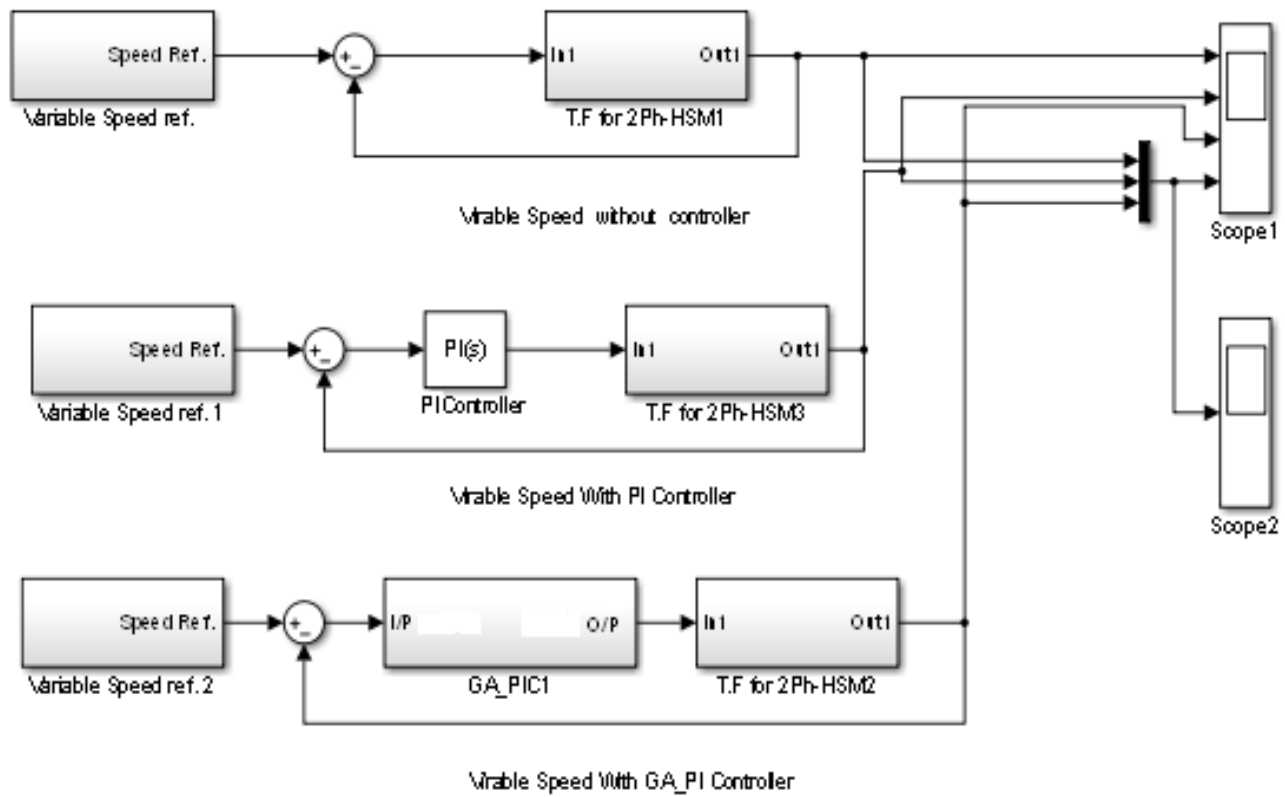

Figure 8 . The simulation model of a 2Ph-HSM system combined for three circuits (without control, with PIC, and with GA_PIC) and for variable speed

\section{CONTROLLING AND OPTIMIZATION FOR THE PLANT}

Many methods of controller and optimization used to improve the work of the plant, the idea still focusing on how to choose the proper method that fit with the working plant which planned to accomplish it. In our work, used the PIC as a controller unit to control for plant and GA_PIC unit to optimize it, the next sections illustrate the two units.

\subsection{The PIC}

Up to now, many of the controllers are PI controllers due to they're soft to grasp [25]. The closed-loop system with a PIC in the direct path appears in Figure 9. The function of PIC is described as:

$$
y(t)=e(t) K_{P}+K_{I} \int_{0}^{t} e(t) d t
$$

according to manual expertise, $K_{P}$ represents the proportional constant gain and $K_{I}$ is the integral constant gain, $e(t)$ represents the error signal between the reference signal $r(t)$ and the plant output $y(t)$, its expounded as $e(t)=r(t)-y(t)$.

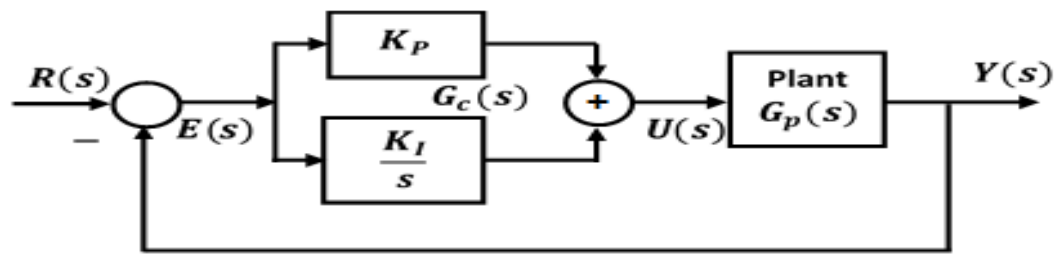

Figure 9. The closed-loop system with a PIC of a plant

The (7) is the transfer function that shows the relation between the PIC input E (s) (error signal) and output $\mathrm{U}(\mathrm{s})$ (input to the plant).

$$
G_{c}(s)=\frac{U(s)}{E(s)}=K_{P}+\frac{K_{I}}{s}
$$


The closed-loop transfer function $G(s)$ is given by (8):

$$
G(s)=\frac{Y(s)}{R(s)}=\frac{G_{c}(s) G_{p}(s)}{1+G_{c}(s) G_{p}(s)}
$$

The adjusting of a PIC consists of choosing gains $K_{P}$ and $K_{I}$ thus that performance specifications are satisfied. The $K_{P}$ parameter effects on the control system as follow: decrease the rise time, increase the overshoot, decrease the error, and make a small change in the turning time, while $K_{I}$ parameter effects as follow: decrease the rise time, increase the overshoot, eliminate the error, and increase the turning time [26].

\subsection{The GA_PIC Optimization}

GA is a heuristic improvement mechanism created by the techniques of natural selection. GA begins with a premier inhabitance including a number of chromosomes, each chromosome appears as a solution to a problem in which its action is evaluated depending on the fitness function. according to the suitability of each individual and its specified probability, a set of chromosomes is chosen to subject three combined operations: selection, crossover, and mutation. The action of these three combined stages authorizes to create new individuals to produce better solutions than the parents which lead to the optimal solution. A fulfillment action of GA_PIC is listed as follows [27].

The best way to implement GA is with a small size for inhabitants in order to permit the PIC to be optimized as fast as potential. The initial inhabitant is created by encryption the PIC parameters, $K_{P}$ and $K_{I}$ to binary series called as a chromosome. The length of a series counts on the wanted accuracy that is about 4 bits. The desired bit series is calculated according to (9).

$$
2^{n-1}<\left(U_{b}-L_{b}\right) \times 10^{4} \leq 2^{n}-1
$$

where $n$ is the No, bits, $U_{b}$ and $L_{b}$ are the upper bounds and lower bounds of PIC parameters.

The evaluation of the suitability of each chromosome by conversion its binary series into real values as PIC parameter values and put it in an objective function known as the fitness function like the mean square error (MSE) and integral absolute error (IAE), the converting operation from binary values to real numbers for each chromosome is done by an encryption process by using (10).

$$
\left.C_{v}=L_{b}+\text { Dec(subseries }\right) \times \frac{\left(U_{b}-L_{b}\right)}{2^{n}-1}
$$

where $C_{v}$ is the real numbers of $K_{P}$ and $K_{I}$, Dec(subseries) represents the decimal values of binary series that comes from (9).

The target of GA is to search for minimum fitness worth, so each group of PIC parameter is moved to the fitness function in order to calculate for a prime fitness value as shown in Figure 10. After calculating the initial fitness values, it goes to the selection operation with its corresponding chromosome. The higher the value of fitness worth, the greater the chance to choose a member of the inhabitant. The tournament selection strategy is a better method due to its present a good choosing strategy able to regulate its selective pressure and inhabitant variety to get better search performance for the GA. In contrast, the strategy of roulette selection that permits to choose feeble chromosomes in numerous times. The chromosomes leave the selection operation toward crossover operation, two mating chromosomes are choosing in a random way, one of them cut-point and another, replace the right section of the two parents to birthing progeny. A one-point crossover is select for basic GA.

The mutation operation comes after crossover operation, it is blocking the algorithm to fall in the local minimum and preserve a diversity of the inhabitance. usually, the lowest mutation rate must be chosen while the greater mutation rate may be changing the searching operation into a random search operation. In the next generation, and after finishing the three operations (selection, crossover, and mutation), the binary series in either chromosome in the inhabitance require to be decryption into real values. A new group of PIC is transmitted to the PIC system to calculate for a new fitness worth. This sequential process passes through the three operations and reiterated until the generations finishing where better fitness is fulfilled. Figure 10 shows the diagram of GA. 


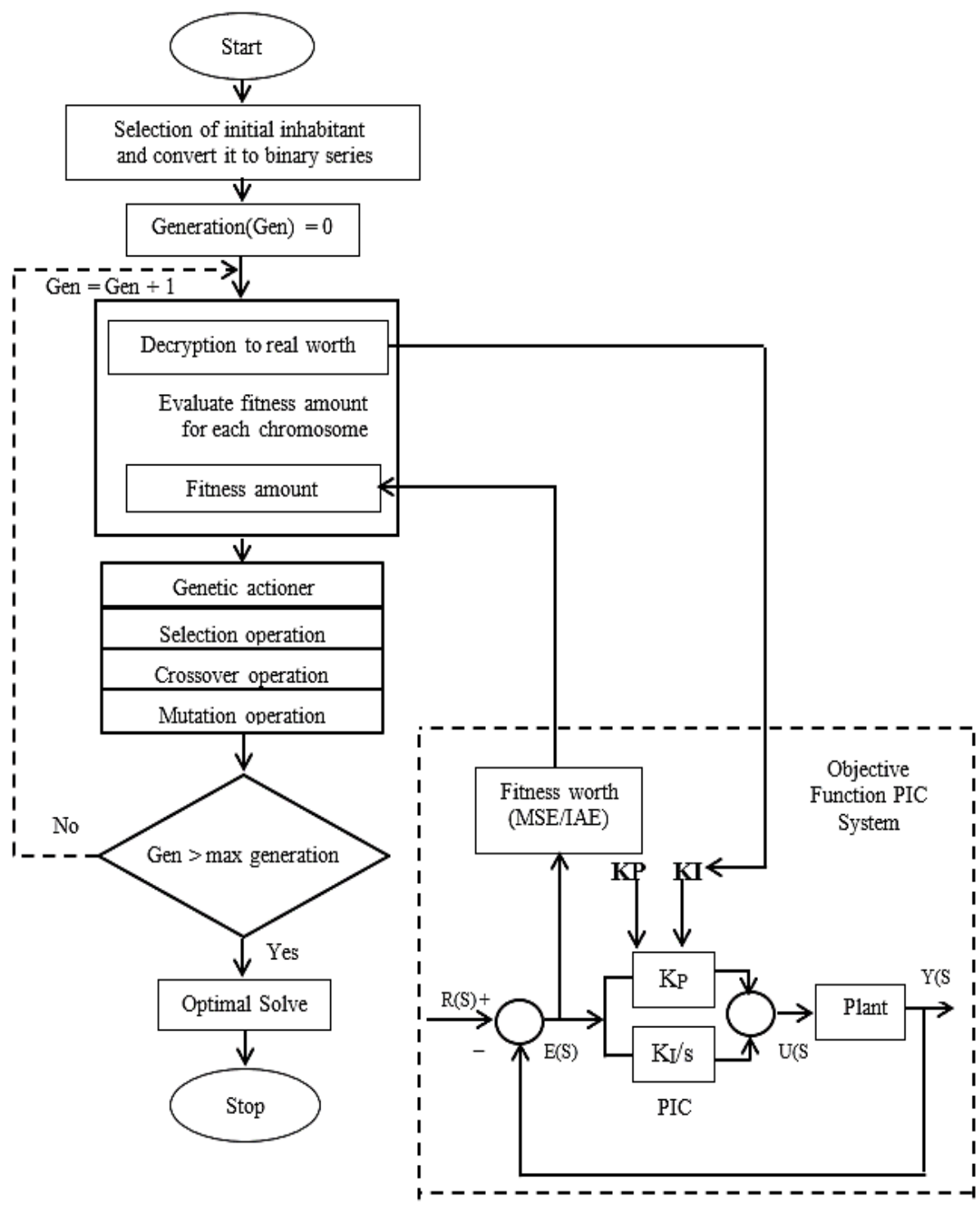

Figure 10. The diagram of GA

\section{THE SIMULATION RESULTS}

In this section, the simulation results discuss through Figures 11-13 which we got from the MATLAB/ SIMULINK program, each figure has two parts (a) and (b): part (a) appears the system response at constant speed, and part (b) appears the system response at variable speed, each part shows the shape of an input signal, output signal, and the comparative between them, the system response is discussed in three cases: without control, with PIC, and with GA_PIC. Figure 11 shows the system response without control at constant and variable speed, the shape appears the response of the system as a worst response compare with the system response with PIC and GA_PIC due to the high values of rising time=1.44, steady state error=6.5.

Figure 12 shows the system response with PIC at constant and variable speed with $K_{P}=16.54$ and $K_{I}=3.86$, the shape appears a good response of the system compare with the system response without controller due to the acceptable values of rising time $=0.09$, steady state error $=0.38$, and overshoot $=8 \%$. Figure 13 shows the system response with GA_PIC optimization at constant and variable speed, the values of KP=14.441 and $\mathrm{KI}=0.032$ at iteration $=65$ as shown in Figure 14, the shape appears the best response of the system compare with the response of system with PIC and without controller due to the good values of rising time $=0.08$, steady state error $=0.28$, and overshoot $=0 \%$. 

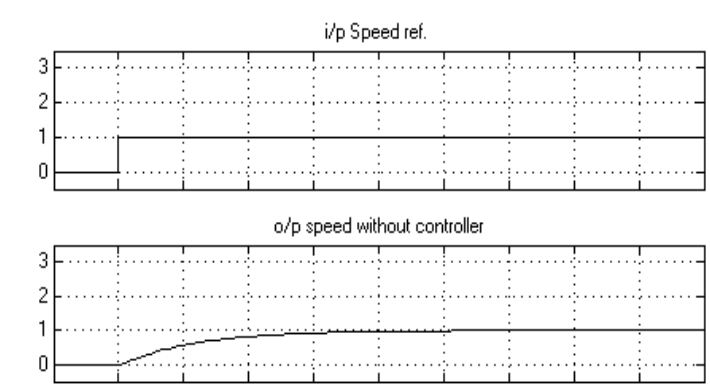

i/p Speed ref. \& $0 / p$ speed without controller

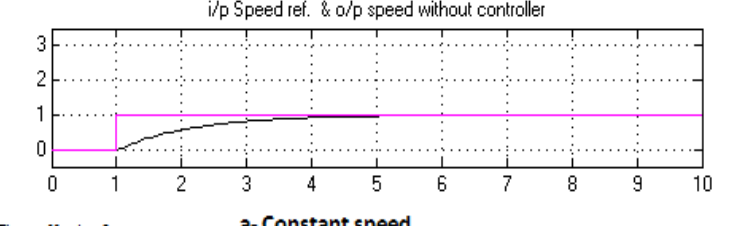

Time offset: 0

a- Constant speed
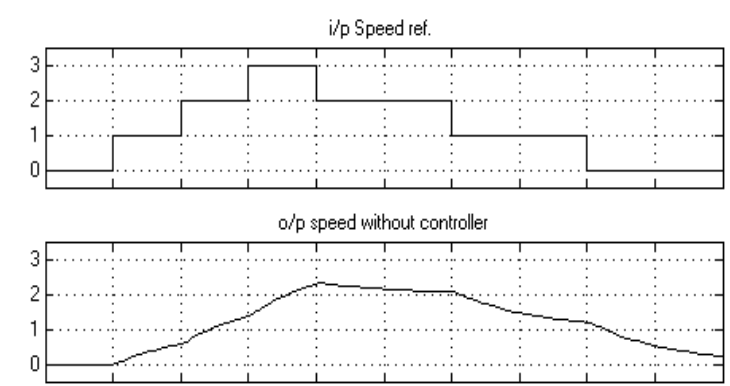

i/p Speed ref. \& o/p speed without controller

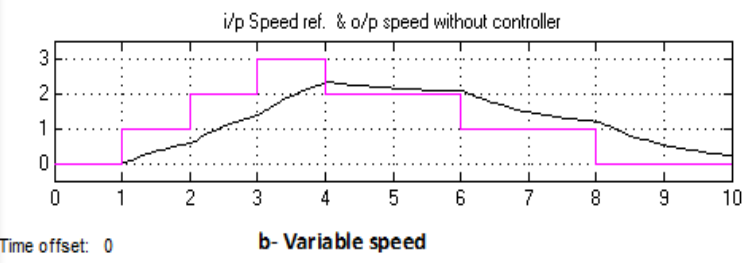

Figure 11. The step response of the system without control at (a) constant speed and (b) variable speed
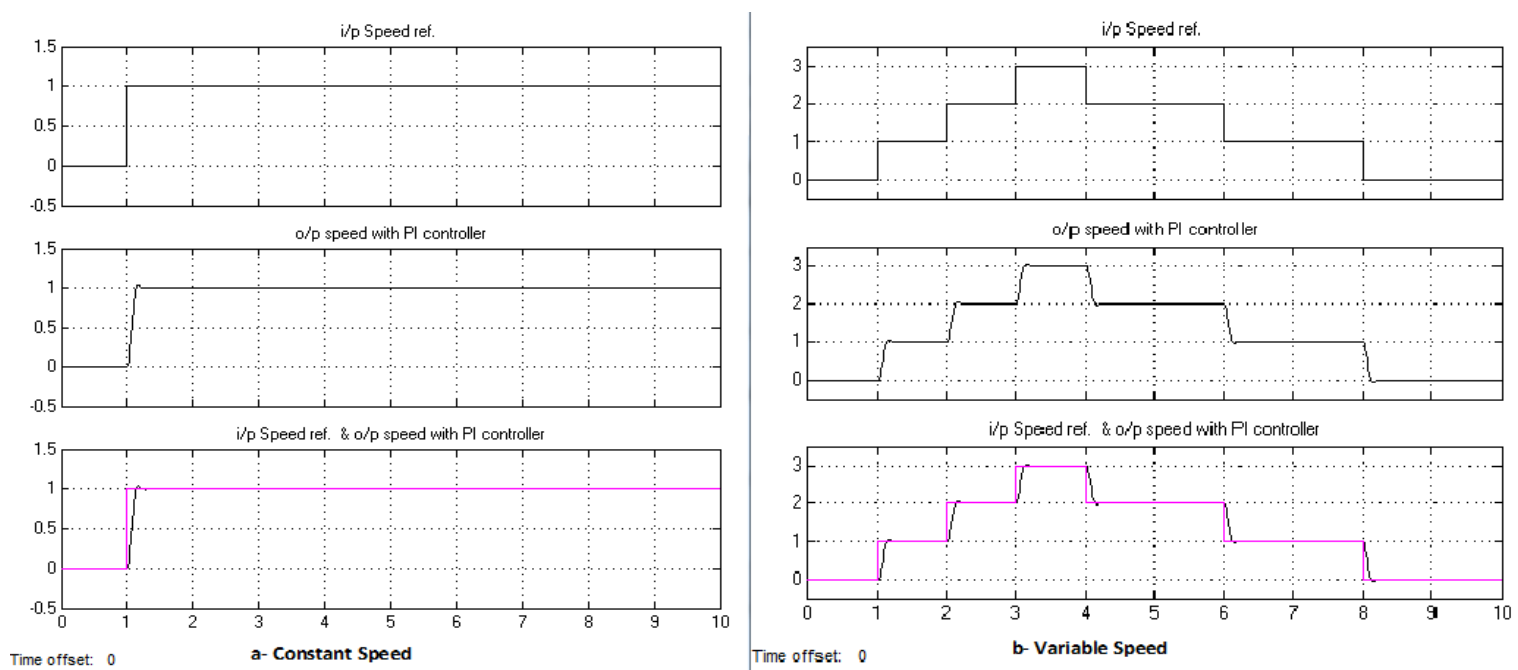

Figure 12. The step response of the system with PIC at (a) constant speed and (b) variable speed
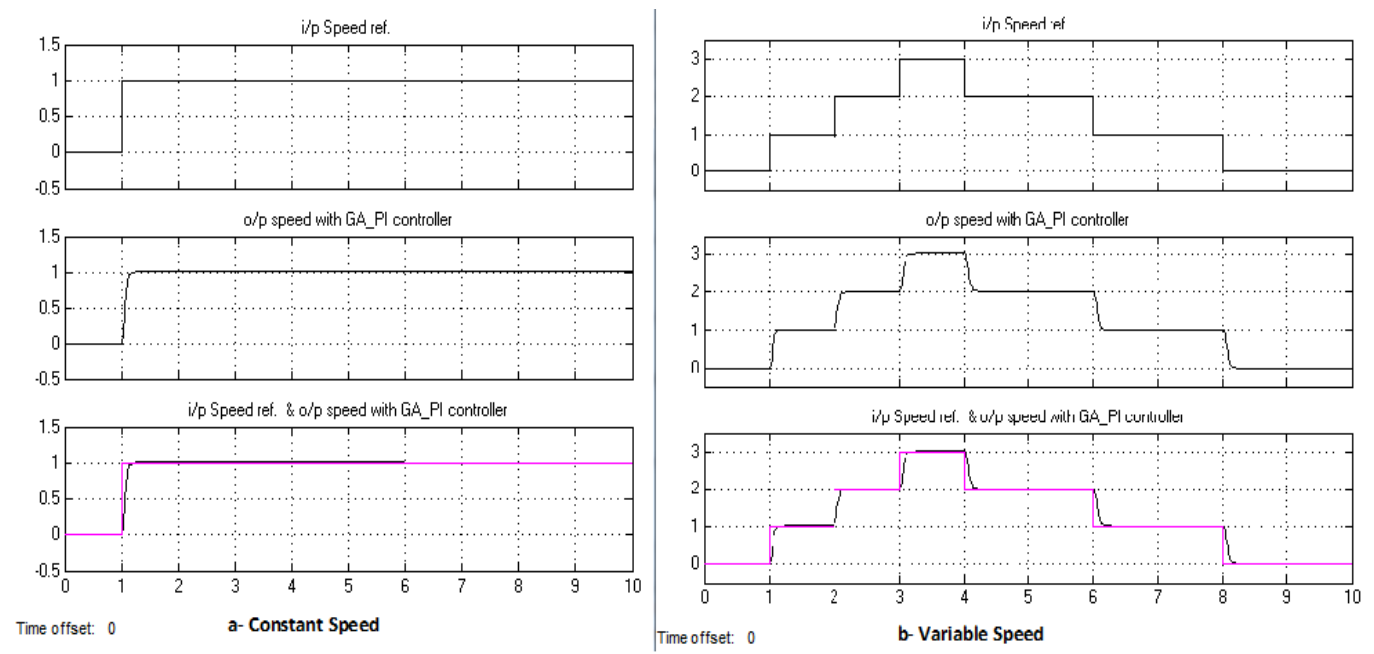

Figure 13. The step response of the system with GA_PIC at (a) constant speed and (b) variable speed 


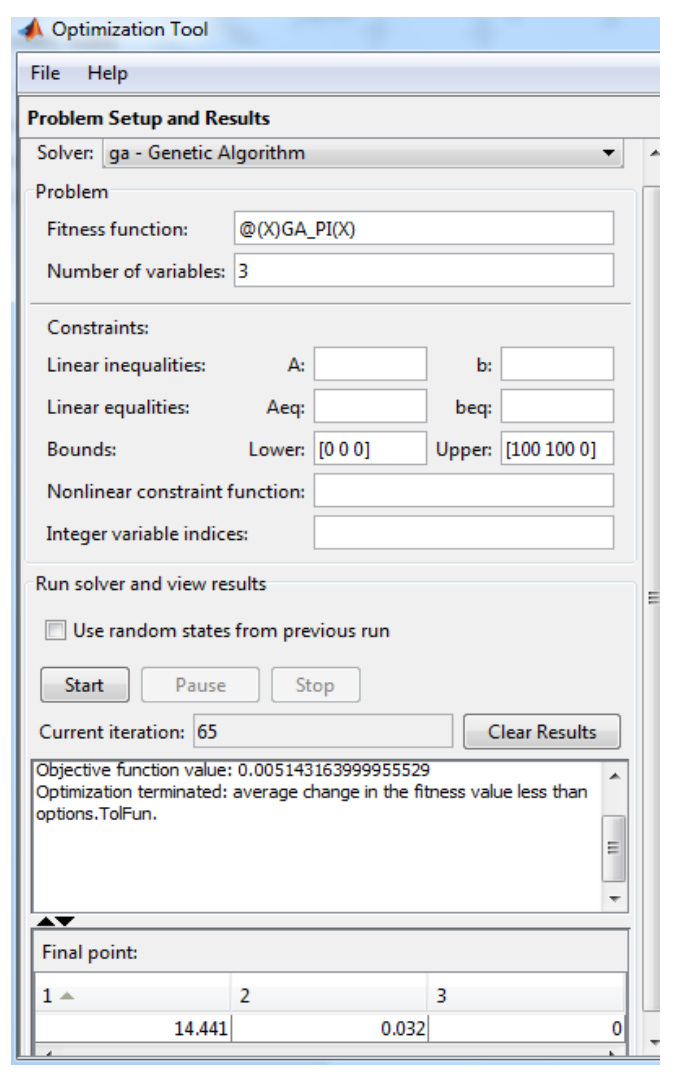

Figure 14. The optimization tools for GA_PIC

\section{CONCLUSIONS AND FUTURE WORKS}

The grinding process is an important process in the manufacturing field especially when using it in difficult environments that the people cannot reach it because of the risks or difficulty working in it, therefore, it becomes necessary to use the robot to overcome those difficulties by controlling this important process by using classical controllers or intelligent controllers such as PIC or GA_PIC. The controlling on the grinding process begin when controlling on grinding wheel to make it adapt with the curved surface through improving the obedient wrist system, this achieves by work of wrist when it changes its position in columnar two trends to adapted the machining surface according to the HS compressive strength which controlled by the SM. So, the grinding wheel becomes more proper to grind the free-shape surfaces.

Whilst the controlling parameters were not proper with the position of the wrist and the controlling system is not able of adjusting the form of the machining surface, the GF can't be controlled at its objective value and the error increases with the movement of the feed. This signifies that the pattern of the surroundings and the obedient wrist system can use in strength controlling when robots grind Free surfaces of the fans with multipoint pieces by a grinding tool. The simulation results of the work are confirming the idea that a GA_PIC has better control performance than the classical PIC due to the GA is to optimize the PIC behavior to get convinced control characteristics. The controlling and simulation of 2Ph-HSM achieved by utilizing the software bundle of the MATLAB program (SIMULINK). The authors suggest using other optimization methods such as fuzzy logic with PIC and particle swarm optimization with PIC to know how they affect the control parameters and compare these methods with GA_PIC to knowledge the best one.

\section{REFERENCES}

[1] Attiya, A. J., Wenyu, Y., \& Shneen, S. W., "PSO_PI Controller of Robotic Grinding Force Servo System," TELKOMNIKA Indonesian Journal of Electrical Engineering, Vol. 15, No. 3, pp. 515-525, September $2015 .$.

[2] Trujillo, O. A., Toro-García, N., \& Hoyos, F. E., "PID controller using rapid control prototyping techniques," International Journal of Electrical \& Computer Engineering, vol. 9, no. 3, pp. 2088-8708, 2019.

[3] Uthman, A., \& Sudin, S., "Antenna Azimuth Position Control System using PID Controller \& State-Feedback Controller Approach," International Journal of Electrical \& Computer Engineering, vol. 8, no. 3, pp. 2088-8708, 2018. 
[4] Kashyap, P. K., \& Kumar, S., "Genetic-fuzzy based load balanced protocol for WSNs," International Journal of Electrical and Computer Engineering, vol. 9, no. 2, pp. 1168, 2019.

[5] Zubeiri, I., Morabit, Y. E., \& Mrabti, F., "Genetic algorithm for vertical handover (GAfVH) in a heterogeneous network," International Journal of Electrical \& Computer Engineering, vol. 9, pp. 2088-8708, 2019.

[6] Gulia, P., "Hybrid swarm and GA based approach for software test case selection," International Journal of Electrical \& Computer Engineering, vol. 9, pp. 2088-8708, 2019.

[7] Wibowo, W. K., \& Jeong, S. K., "Genetic algorithm tuned PI controller on PMSM simplified vector control," Journal of Central South University, vol. 20, no. 11, pp. 3042-3048, 2013.

[8] Li, W. L., Xie, H., Zhang, G., Yan, S. J., \& Yin, Z. P., “3-D shape matching of a blade surface in robotic grinding applications," IEEE/ASME Transactions on Mechatronics, vol. 21, no. 5, pp. 2294-2306, 2016.

[9] Wang, J., Zhang, G., Zhang, H., \& Fuhlbrigge, T., "Force control technologies for new robotic applications," In 2008 IEEE International Conference on Technologies for Practical Robot Applications, pp. 143-149. IEEE. 2008.

[10] Thangaraj, R., Chelliah, T. R., Pant, M., Abraham, A., \& Grosan, C., "Optimal gain tuning of PI speed controller in induction motor drives using particle swarm optimization," Logic Journal of the IGPL, vol. 19, no. 2, pp. 343-356, 2011.

[11] Bekakra, Y., \& Attous, D. B., "Optimal tuning of PI controller using PSO optimization for indirect power control for DFIG based wind turbine with MPPT," International Journal of System Assurance Engineering and Management, vol. 5, no. 3, pp. 219-229, 2014.

[12] Reeves, C. R., "Modern heuristic techniques for combinatorial problems, advanced topics in computer science," Modern Heuristic Techniques for Combinatorial Problems: Advanced Topics in Computer Science, 1995.

[13] Zhang, S. Y., \& Wang, X. M., "Study of fuzzy-PID control in MATLAB for two-phase hybrid stepping motor, In Applied Mechanics and Materials," Trans Tech Publications, vol. 341, pp. 664-667, 2013.

[14] Attiya, A. J., Wenyu, Y., \& Shneen, S. W., “Compared with PI, Fuzzy_PI \& PSO_PI Controllers of Robotic Grinding Force Servo System," TELKOMNIKA Indonesian Journal of Electrical Engineering, vol. 16, no. 1, pp. 65-74, 2015.

[15] Chopra, V., Singla, S. K., \& Dewan, L., "Comparative analysis of tuning a PID controller using intelligent methods, ACTA Polytechnica hungarica," vol. 1, no. 8, pp. 235-249, 2014.

[16] Malhotra, R., Singh, N., \& Singh, Y., "Genetic algorithms: concepts, design for optimization of process controllers," Computer and Information Science, vol. 4, no. 2, pp. 39, 2011.

[17] Saad, M. S., Jamaluddin, H., \& Darus, I. Z., "PID controller tuning using evolutionary algorithms," Wseas transactions on Systems and Control, vol. 7, no. 4, pp. 139-149, 2012.

[18] ZC Li, B Lin, YS Xu, J Hu., "Experimental studies on grinding forces and force ratio of the unsteady state grinding technique," Journal of Materials Processing Technology, vol. 129, no.76-80, 2002.

[19] Nguyen TD, Tseng KJ, Zhang, S, Nguyen HT., "A Novel Axial Flux Permanent-Magnet Machine for Flywheel Energy Storage System Design and Analysis," IEEE Transactions on Industrial Electronics, vol. 58, no. 9, pp. 3784-3794, 2011.

[20] Zhang Tuanshan, Zhang Na, Wu Yuting., "Study of driving bipolar stepper motors based on enhanced STM32," Electronic Measurement Technology, vol. 33, no. 10, 16-18, 2010.

[21] Andrew J Blauch, Marc Bodson, John Chiasson., "High-speed Parameter Estimation of Stepper," Motors IEEE Transactions on control systems technology, vol. 1, no. 4, 270-279, 1993.

[22] Attiya, A. J., Wenyu, Y., \& Shneen, S. W., "Fuzzy-PID Controller of Robotic Grinding Force Servo System," International Journal of Electrical and Computer Engineering (IJECE), vol. 15, no. 1, pp. 87-99, 2015.

[23] ZAKY, M. S., ISMAEIL, E. M., \& KHATER, M. M., "DSP-based real-time control of a two-phase hybrid stepping motor," ACTA Electrotechnica, vol. 51, no. 2, 109-113, 2010.

[24] Attiya, Adnan Jabbar, Yang Wenyu, and Salam Waley Shneen., "Compared with PI, Fuzzy_PI \& PSO_PI Controllers of Robotic Grinding Force Servo System,” TELKOMNIKA Indonesian Journal of Electrical Engineering, vol. 16, no. 1, pp. 65-74, 2015.

[25] Cheng-Tsung Lin, Chung-Wen Hung and Chih-Wen Liu., "Fuzzy PI controller for BLDC motors considering Variable Sampling Effect," IECON 2007 - 33rd Annual Conference of the IEEE Industrial Electronics Society, Taipei, Taiwan, Nov. 5-8, 2007.

[26] Amaral, J. M., Tanscheit, R., \& Pacheco, M. A., "Tuning PID controllers through genetic algorithms," Complex Systems, vol. 2, no. 3, 2018.

[27] Shneen, Salam Waley, Chengxiong Mao, and Dan Wang., "Advanced optimal PSO, Fuzzy and PI controller with PMSM and WTGS at $5 \mathrm{~Hz}$ side of generation and $50 \mathrm{~Hz}$ Side of Grid," International Journal of Power Electronics and Drive Systems, vol. 7, no. 1, pp. 173, 2016. 